\title{
Einführung: Aus grün mach' grau, zäun' ein und bau! \\ Zur Steuerung der Flächeninanspruchnahme
}

Dem im Titel genannten Credo folgen auch solche Gemeinden seit Jahrzehnten, deren

Bevölkerungszahl inzwischen zurückgeht. Eine neue Diskussion über die Rolle und das Zusammenspiel ökonomischer und planerischer Instrumente in der Raumplanung könnte das Problembewußtsein schärfen und innovativen Steuerungsinstrumenten den Weg bahnen.
Irmi Seidl, Barbara Schultz

Gastherausgeberinnen des Schwerpunkts
D ie hohe Flächenneuinanspruchnahme ist ein europaweites Problem (EEA 2005). In den deutschsprachigen Ländern geht sie vor allem vom Wohnungsbau aus. So hat in der Schweiz die Siedlungs- und Verkehrsfläche zwischen 1979/85 und 1992/97 um 13,3 Prozent zugenommen - die für Wohnzwecke aber um 25,4 Prozent (BFS 2001). In Österreich und Deutschland sind ähnliche Trends erkennbar (BEV o.J., Statistisches Bundesamt 2005).

Diese Entwicklung hat zwei zentrale Ursachen: Erstens führen demographische sowie gesellschaftliche Trends - kleinere und mehr Single-Haushalte, höherer individueller Wohnflächenanspruch und höherer Lebensstandard - zu einer verstärkten Nachfrage nach Ein- und Zweifamilienhäusern und generell nach größerer Wohnfläche pro Kopf. Zweitens bestehen kaum Anreize, die Flächeninanspruchnahme zu begrenzen (RSU 2000): Für Bauherren bedeutet flächenschonendes Bauen oft Einschränkungen und höhere Kosten - etwa durch mehrstöckigen statt Flachbau. Die Gemeinden sehen sich in einem harten Wettbewerb um neue Einwohner(innen), neue Investitionen und zusätzliches kommunales Steueraufkommen. Entsprechend entscheiden sie sich im Rahmen ihrer Planungshoheit und im Zuge der planerischen Abwägung meist für die Ausweisung von Bauland und großzügige Baugenehmigungen statt für eine restriktive Flächenpolitik. Die regionalen Planungsinstitutionen scheuen sich häufig, Gemeinden bei der Baulandausweisung einzuschränken. Den letztlich im Paradigma des Wachstums gründenden Argumenten der Gemeinden will kaum jemand widersprechen. Vor diesem Hintergrund stoßen Forderungen nach geringerer Flächeninanspruchnahme und dem Einsatz wirksamerer Instrumente auf Nichtbeachtung, Ablehnung und Widerstand.

\section{Das verkannte Problem}

Den genannten Kräften etwas entgegenzusetzen, ist auch deshalb schwierig, weil für das Individuum die negativen Wirkungen von Überbauung und Zersiedelung auf den ersten Blick kaum wahrnehmbar sind. Aus Sicht des einzelnen beansprucht das eigene
Bauvorhaben nicht viel Fläche; es erscheint harmlos und "unschuldig" (Schmalholz 2002). Es ist die Summe der Eingriffe, die die Folgen zu Problemen macht: Landschaftszerschneidung, Verlust fruchtbarer Böden, Beeinträchtigung des Wasserhaushaltes, des Meso- und Mikroklimas sowie des Landschaftsbilds, Gefährdung der Biodiversität, Verlärmung, Zersiedelung und resultierende Kosten für das Gemeinwesen (zum Beispiel Häberli et al. 1991, Baur et al. 2005, Jaeger et al. 2005).

Die Raumplanung fordert weniger Flächeninanspruchnahme. Ernsthaft unterstützt wird sie darin lediglich vom Landschafts-, Umwelt- und Naturschutz. Dabei führen auch diese Kreise noch einen lückenhaften Diskurs: Sie widmeten sich bisher mehr den stofflichen Bodenbelastungen als der quantitativen Flächeninanspruchnahme. Sie betonten die negativen Konsequenzen der Versiegelung, thematisieren aber erst seit kurzem die Infrastrukturfolgekosten und die ökologischen Folgen von Zersiedelung und Zerschneidung. Lange Zeit wurde das demographische und wirtschaftliche Wachstum fast als Lebensgesetz angesehen; dagegen werden die stagnierenden oder zurückgehenden Wachstums- und Bevölkerungszahlen noch kaum wahrgenommen (Siedentop 2005). Den Diskurs um die genannten Aspekte zu erweitern, ist Voraussetzung für ein größeres gesellschaftliches Bewußtsein über das Problem der Flächeninanspruchnahme.

\section{Lösungsansätze}

Die hohe Flächeninanspruchnahme wird verschiedentlich mit einem Versagen raumplanerischer Instrumente erklärt (Gmünder
Kontakt: PD Dr. Irmi Seidl | Eidgenössische Forschungsanstalt WSL Zürcherstr. 111 | CH-8903 Birmensdorf | Tel.: +41 447392324 | Fax: +41447392588 | E-Mail: irmi.seidl@wsl.ch

Barbara Schultz, Geographin M.A. | Institut für Raumund Landschaftsentwicklung | ETH Zürich CH-8093 Zürich | E-Mail: schultz@nsl.ethz.ch 
2004). Doch in der Tat liegt häufig politisches Versagen vor (Bertschi 2002, Einig 2006, in diesem Heft). Um dieses auszugleichen, ist die Einführung umweltökonomischer Instrumente im Gespräch (zum Beispiel Bizer und Lang 1997, Bergmann et al. 1999, ARE 2003). Damit erhalten einerseits seit langem diskutierte Instrumente wie die staatliche Abschöpfung planerisch geschaffener Mehrwerte auf Grundstücken (Plattner 1992) oder die Versiegelungsabgabe (Bizer und Truger 1996) neue Aufmerksamkeit. Andererseits werden neue Instrumente wie Flächenzertifikate (Wegelin 2006, Walz 2006, Zollinger 2006, alle in diesem Heft) geprüft. Ökonomische Instrumente haben - im Gegensatz zu vielen planerischen Instrumenten - den Vorteil, daß gesetzte Ziele in Verwaltungs- und Abwägungsprozessen nur geringfügig oder gar nicht aufgeweicht werden können. Aufgabe der Raumplanung und der Ökonomie ist es, umsetzungsfähige Konzepte für die Anwendung ökonomischer Instrumente zu entwickeln und mit Planungsinstrumenten zu verknüpfen. Daß die Raumplanung den Rahmen für die Flächennutzung vorgeben muß, innerhalb dessen die ökonomischen Instrumente angewendet werden können, darüber herrscht sowohl auf Seite der Ökonom(inn)en wie auch der Raumplaner(innen) weitgehend Konsens.

Ein Workshop zum Thema „Steuerung der Flächeninanspruchnahme mit Markt und Staat"1 gab den Anstoß für diesen Schwerpunkt: Zunächst zeigt Rainer Walz die Vorteile ökonomischer Instrumente in der Raumplanung und diskutiert die erreichbaren Ziele. Er erläutert die Probleme von Flächenkontingenten angesichts der Eigenarten des Gutes „Boden“. Eine seiner Schlußfolgerungen: Die offenen Fragen zu Flächenzertifikaten ähneln jenen zu den nun etablierten $\mathrm{CO}_{2}$-Zertifikaten vor einigen Jahren. | Fritz Wegelin stellt Erkenntnisse aus dem Schweizer Raumentwickungsbericht 2005 (ARE 2005) vor und begründet damit sein Plädoyer für die Einführung von Flächennutzungszertifikaten in der Schweiz. Gesellschaftlich und ökonomisch akzeptable Varianten von Zertifikaten seien möglich. | Fritz Zollinger benennt Potentiale und Grenzen handelbarer Flächenzertifikate in der Schweiz. | Klaus Einig beschreibt, warum Gemeinden in Deutschland planerische Instrumente oft ignorieren. Als treibende Kräfte für die hohe Flächeninanspruchnahme nennt er Föderalismus, Steuerwettbewerb und Wachstumsparadigma. | Barbara Schultz und Sabine Friedrich identifizieren als eine Ursache für die hohe Flächeninanspruchnahme die für Gemeinden mangelnde Transparenz bei den Infrastrukturkosten. Sie stellen ein Modell vor, mit dem Gemeinden die langfristigen Infrastrukturkosten ihrer baulichen Entwicklung berechnen können. | Positionen gesellschaftlicher Interessengruppen verdeutlichen die Interviews mit der Schweizer Ständerätin und Präsidentin der Stiftung Landschaftsschutz Schweiz, Erika Forster-Vannini, sowie mit Sandra Burlet, Vertreterin des Hauseigentümerverbandes Schweiz.

1 Der Workshop fand am 16.03.2006 an der ETH Zürich statt. Veranstaltet wurde er vom Institut für Raum- und Landschaftsentwicklung der ETH Zürich, der Eidgenössischen Forschungsanstalt WSL sowie dem Schweizerischen Bundesamt für Raumentwicklung (ARE). Die Referate sind abrufbar unter http://lep.ethz.ch/index.php?id=455.

\section{Literatur}

ARE (Bundesamt für Raumentwicklung). 2003. Marktwirtschaftliche Instrumente in der Raumplanung. ExpertInnen-Workshop. Dokumentation. Bern: ARE.

ARE (Bundesamt für Raumentwicklung) 2005. Raumentwicklungsbericht 2005. Bern: ARE.

ARE (Bundesamt für Raumentwicklung). 2006. Flächennutzungszertifikate. Systemdesign und wirtschaftliche Auswirkungen. Bern: ARE.

Baur, B. et al. 2004. Biodiversität in der Schweiz: Zustand, Erhaltung, Perspektiven. Grundlagen für eine nationale Strategie. Bern: Haupt.

Bergmann, A., K. Einig, G. Hutter, B. Müller, S. Siedentop (Hrsg.). 1999. Siedlungspolitik auf neuen Wegen. Steuerungsinstrumente für eine ressourcenschonende Flächennutzung. Berlin: edition sigma.

Bertschi, M. 2002. Massengräber für gute Vorsätze. tec21 2002/26: 19-24.

Bizer, K., J. Lang. 1997. Ansätze für ökonomische Anreize zum sparsamen und schonenden Umgang mit Bodenflächen. Berlin: Umweltbundesamt.

Bizer, K., A. Truger. 1996. Die Steuerung der Bodenversiegelung durch Abgaben. Zeitschrift für angewandte Umweltforschung 9/3: 379-389.

BEV (Bundesamt für Eich- und Vermessungswesen). o. J. Daten der Regionalinformation der Grundstücksdatenbank 1999-2004. Wien: BEV.

BFS (Bundesamt für Statistik). 2001. Bodennutzung im Wandel. Arealstatistik Schweiz. Neuchâtel: BFS.

Einig, K. 2006. Von der Kunst des Kuchenteilens. Die Umsetzung des Flächenreduktionsziels in Deutschland. GAIA 15/3: 185-186.

EEA (European Environment Agency). 2005. The European environment State and outlook 2005. Kopenhagen: EEA.

Gmünder, M. 2004. Mit weniger Planung zu mehr Raum? Zum Einsatz anreizorientierter Instrumente in der Raumplanung. In: Perspektiven der Wirtschaftspolitik. Herausgegeben von C. Schaltegger, S. Schaltegger. Zürich: vdf. 557-570.

Häberli, R., C. Lüscher, B. Praplan Chastonay, C. Wyss. 1991. Bodenkultur. Vorschläge für eine haushälterische Nutzung des Bodens in der Schweiz. Zürich: vdf.

Jaeger, J., S. Grau, W. Haber (Hrsg.). 2005. Schwerpunkt: Landschaftszerschneidung. GAIA 14/2: 98-185.

Plattner, R. M. 1992. Das Modell Basel: Mehrwert abschöpfen, Minderwert abgelten. Raum. Österreichische Zeitschrift für Raumplanung und Regionalpolitik 1992/März: 30-31.

RSU (Rat von Sachverständigen für Umweltfragen) 2000. Umweltgutachten 2000. Stuttgart: Metzler-Poeschel.

Schmalholz, M. 2002. Zur rechtlichen Zulässigkeit handelbarer Flächenausweisungsrechte. Zeitschrift für Umweltrecht Sonderheft 2002: 158-163.

Siedentop, S. 2005. Problemdimensionen der Flächeninanspruchnahme. In: Flächensparbuch. Herausgegeben vom Institut für Stadt- und Regionalplanung der TU Berlin. ISR Diskussionsbeiträge 56: 19-27.

Statistisches Bundesamt. 2005. Umweltstatistische Erhebungen. Bodennutzung nach wirtschaftlichen Aktivitäten. www.destatis.de/basis/d/umw/ ugrtab9.php (abgerufen 11.08.2006).

Walz, R. 2006. Instrumente der Flächenpolitik. Eine Einordnung in generelle Trends der umweltpolitischen Instrumentediskussion. GAIA 15/3: 177-180.

Wegelin, F. 2006. Ökonomische Instrumente unterstützen die Raumplaung. Das Bundesamt für Raumentwicklung zur Verringerung der Flächeninanspruchnahme in der Schweiz. GAIA 15/3: 181-183.

Zollinger, F. 2006. Potential und Grenzen handelbarer Flächenzertifikate in der Schweiz. GAIA 15/3: 184.

Irmi Seidl

Geboren 1962 in Holzkirchen, Bayern. Studium der Wirtschaftswissenschaften. 1993 Promotion an der Universität St. Gallen, 2003 Habilitation an der Universität Zürich. Seit 2002 an der Eidgenössischen Forschungsanstalt WSL in Birmensdorf, seit Juli 2006 Leiterin a. i. der Forschungseinheit Wirtschafts- und Sozialwissenschaften.

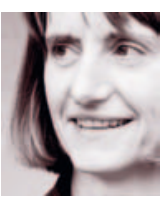

Die Kurzbiographie von Barbara Schultz finden Sie auf Seite 189 